Proyecciones

Vol. 19, No 3, pp. 227-248, December 2000.

Universidad Católica del Norte

Antofagasta - Chile

DOI: 10.4067/S0716-09172000000300002

\title{
SUR LE SPECTRE D'UN OPÉRATEUR QUASILINÉAIRE ELLIPTIQUE "DÉGÉNÉRÉ"
}

\author{
A. ANANE, A. BENAZZI and O. CHAKRONE \\ UNIVERSITÉ MOHAMED I, MAROC
}

\begin{abstract}
We study an eigenvalue problem involving an operator $\mathcal{A}_{p}^{0}$ that generalizes the pseudo-Laplacian $\Delta_{p}^{0}$ and the p-Laplacian. In particular, we will prove the isolation and simplicity of the first eigenvalue. We will treat with more details the particular case of $\Delta_{p}^{0}$ on a domaine $\Omega$ which is a cube.
\end{abstract}




\section{Introduction}

Nous nous intéressons à l'étude des valeurs propres $\lambda$ et des fonctions propres associées $u$ solutions du problème :

$$
\begin{aligned}
(V P) & \left\{\mathcal{A}_{p}^{0} u=-\sum_{i, k, l=1}^{N} \frac{\partial}{\partial x_{1}}\left[\left(\sum_{r, s=1}^{N} a_{r s}^{i}(x) \frac{\partial u}{\partial x_{r}} \frac{\partial u}{\partial x_{s}}\right)^{\frac{p-2}{2}} a_{k}^{i}(x) \frac{\partial u}{\partial x_{k}}\right]\right. \\
=\lambda m(x)|u|^{p-2} u & \quad \operatorname{dans} \quad \Omega \\
u=0 & \text { sur } \quad \partial \Omega
\end{aligned}
$$

où $\Omega$ est un domaine borné de $\mathbf{R}^{N}, 1<p<\infty, m$ est une fonction poids et les $a_{k l}^{i} \in L^{\infty}(\Omega)$ sont des fonctions données. L'opérateur $A_{p}^{0}$ est une généralisation du $p$-Laplacien $\Delta_{p}$ et du pseudo-Laplacien

$$
\Delta_{p}^{0} u=\sum_{i=1}^{N} \frac{\partial}{\partial x_{i}}\left(\left|\frac{\partial u}{\partial x_{i}}\right|^{p-2} \frac{\partial u}{\partial x_{i}}\right)
$$

Nous montrons l'existence d'une suite de valeurs propres qui tend vers l'infini, l'isolation et la simplicité de la première valeur propre.

Des travaux analogues ont été fait par Anane [1] pour l'opérateur $\Delta_{p}$ en 1987 contrairement au pseudo-Laplacien qui n'a jamais fait l'objet d'une telle étude en particulier, et l'opérateur $\mathcal{A}_{p}^{0}$ de manière générale. Signalons également l'amélioration apportée par Linqdvist sur le domaine pour l'étude de la simplicité de la première valeur propre de $\Delta_{p}$.

\section{Préliminaires}

\subsection{Définition de l'opérateur $\mathcal{A}_{p}^{0}$}

Nous définissons l'opérateur $\mathcal{A}_{p}^{0}$ sur $W_{0}^{1, p}(\Omega)$ par :

$$
\mathcal{A}_{p}^{0} u=-\sum_{i, k, l=1}^{N} \frac{\partial}{\partial x_{l}}\left[\left(\sum_{r, s=1}^{N} a_{r s}^{i}(x) \frac{\partial u}{\partial x_{r}} \frac{\partial u}{\partial x_{s}}\right)^{\frac{p-2}{2}} a_{k}^{i}(x) \frac{\partial u}{\partial x_{k}}\right]
$$


où les $a_{k l}^{i}$ sont des fonctions $L^{\infty}(\Omega)$ telles que :

$$
a_{k l}^{i}(x)=a_{l k}^{i}(x), \quad \text { pour } i, k, l=1, \ldots, N
$$

de sorte que pour $i=1, \ldots, N$ la forme bilinéaire

$$
a_{i}\left(\xi, \xi^{\prime}\right)=\sum_{k, l}^{N} a_{k l}^{i}(x) \xi_{k} \cdot \xi_{l}^{\prime}, \xi, \xi^{\prime} \in \mathbf{R}
$$

soit symétrique.

Il est clair que l'opérateur $\mathcal{A}_{p}^{0}$ est bien défini sur $W_{0}^{1, p}(\Omega)$ à valeurs dans $W^{-1, p^{\prime}}(\Omega)$.

Nous supposons dans toute la suite que pour tout $i=1, \ldots, N$

$$
a_{i}(\xi, \xi) \geq\left|\xi_{i}\right|^{2}, \quad \text { pour tout } \xi \in \mathbf{R}^{N}
$$

où $\xi_{i}$ est la $i^{\text {ème }}$ composante de $\xi$.

Nous noterons pour tout $i=1, \ldots, N, a_{k l}^{i}(x):=a_{k l}^{i}$ et $a_{i}(\xi, \xi):=$ $|\xi|_{i}^{2}$

$|\cdot|_{i}$ est une semi-norme sur $\mathbf{R}^{N}$ ainsi pour tout $u$ et tout $v$ dans $W^{-1, p^{\prime}}(\Omega)$

$$
\left\langle\mathcal{A}_{p}^{0} u, v\right\rangle=\sum_{i, k, l=1}^{N} \int_{\Omega}|\nabla u|_{i}^{p-2} a_{k l}^{i} \frac{\partial u}{\partial x_{k}} \frac{\partial v}{\partial x_{l}} d x
$$

Quand $a_{k l}^{i}=\delta_{i, k, l} \mathcal{A}_{p}^{0}=\Delta_{p}^{0}$, quand $a_{k l}^{i}=\frac{1}{N} \delta_{k, l} \mathcal{A}_{p}^{0}=\Delta_{p}$, et quand $a_{k l}^{i}=\frac{1}{N} a_{k l} \mathcal{A}_{p}^{0}=\mathcal{A}_{p}, \delta$ réfère au symbole de Kronecker.

Remarque 1. Nous pouvons facilement vérifier que

$$
\left[\left(\left\langle\mathcal{A}_{p}^{0} u, v\right\rangle\right)^{1 / p}=\left(\int_{\Omega} \sum_{i=1}^{N}|\nabla u|_{i}^{p} d x\right)^{1 / p}\right]
$$

est une norme équivalente à la norme $W_{0}^{1, p}(\Omega)$. Nous noterons cette norme par $\|.\|_{\mathcal{A}_{p}}$ et que $\left(W_{0}^{1, p}(\Omega),\|\|.\right)$ est convexe uniformément. 


\subsection{Propriétés élémentaires de $\mathcal{A}_{p}^{0}$}

i) L'opérateur $\mathcal{A}_{p}^{0}$ est un homéomorphisme de $W_{0}^{1, p}(\Omega) \operatorname{sur} W^{-1, p^{\prime}}(\Omega)$.

ii) $\mathcal{A}_{p}^{0}$ est strictement monotone dans le sens suivant :

Pour $u \neq v \in W_{0}^{1, p}(\Omega),\left\langle\mathcal{A}_{p}^{0} u-\mathcal{A}_{p}^{0} v, u-v\right\rangle$ est strictement positif.

iii) $\mathcal{A}_{p}^{0}$ est coercif dans le sens suivant :

$$
\lim _{\|u\|_{\mathcal{A}_{p} \rightarrow+\infty}} \frac{\left\langle\mathcal{A}_{p}^{0} u, u\right\rangle}{\|u\|_{\mathcal{A}_{p}}}=+\infty
$$

La démonstration de ces résultats est une adaptation des méthodes utilisées pour le traitement des opérateurs variationnels (cf. [5]

iv) Considérons la fonctionnelle suivante $: \mathcal{A}_{p}: W_{0}^{1, p}(\Omega) \rightarrow \mathbf{R}$ définie par

$$
\mathcal{A}_{p}(u)=\frac{1}{p} \int_{\Omega} \sum_{i=1}^{N}|\nabla u|_{i}^{p} d x
$$

Alors $\mathcal{A}_{p}^{0}(u)$ n'est autre que la dérivée au sens de Fréchet de $\mathcal{A}_{p}$.

$$
\mathcal{A}_{p}^{\prime}(u)=\mathcal{A}_{p}^{0}(u)
$$

donc $\mathcal{A}_{p}^{0}$ dérive d'un potentiel.

\section{Existence d'une suite de valeurs propres qui tend vers l'infini}

Dans cette section nous montrons qu'il existe une suite de valeurs propres qui tend vers l'infini par la méthode de Ljusternik-Schnirelman et en utilisant le théorème fondamental de multiplicité.

\subsection{Préliminaires}

Définissons la fonctionnelle $\Phi$ de la manière suivante :

$$
\Phi(u)=\left[\mathcal{A}_{p}(u)\right]^{2}-B(u)
$$


où

$$
B(u)=\frac{1}{p} \int_{\Omega} m|u|^{p} d x
$$

Il est clair que $\mathcal{A}_{p}$ et $B$ sont de classe $\mathcal{C}^{1}$ sur $W_{0}^{1, p}(\Omega)$. Leurs dérivées respectives en un point $u \in W_{0}^{1, p}(\Omega)$ sont

$$
\mathcal{A}_{p}^{\prime}(u)=\mathcal{A}_{p}^{0}(u)
$$

et

$$
B^{\prime}(u)=m|u|^{p-2} u
$$

et $\Phi$ est paire et de classe $\mathcal{C}^{1}$ sur $W_{0}^{1, p}(\Omega)$.

Nous supposons que

$$
\operatorname{mes}(\{x \in \Omega ; m(x)>0\}) \neq 0
$$

Et nous nous intéressons aux solutions faibles du problème (V.P.) défini par :

$$
(V . P .)\left\{\begin{array}{l}
(\lambda, u) \in \mathbf{R}_{+} \times W_{0}^{1, p}(\Omega) \\
\sum_{i, k, l=1}^{N} \int_{\Omega}|\nabla u|_{0}^{p-2} a_{k l}^{i}(x) \frac{\partial u}{\partial x_{k}} \frac{\partial v}{\partial x_{l}} d x=\lambda \int_{\Omega} m|u|^{p-2} u v d x \\
\text { pour tout } v \text { appartenant à } W_{0}^{1, p}(\Omega)
\end{array}\right.
$$

Definition 1. Nous dirons que $\lambda$ est une valeur propre, s'il existe une fonction $u$ non nulle de $W_{0}^{1, p}(\Omega)$ telle que le couple $(\lambda, u)$ soit une solution de (V. P.). $u$ sera dite une fonction propre associée à la valeur propre $\lambda$.

\subsection{Existence d'une suite de valeurs propres qui tend vers l'infini}

Sous l'hypothèse 3.1, nous montrons qu'il existe une suite de valeurs propres qui tend vers l'infini.

La démonstration est analogue à celle donnée par Anane dans [1]. Nous transformons le problème aux valeurs propres et fonctions propres en un problème aux points critiques et valeurs critiques.

Nous vérifions facilement que si $u \neq 0$ est un point critique de $\phi$ et $c$ est la valeur critique associée, alors $\lambda=\frac{1}{2 \sqrt{-c}}$ est une valeur propre et $u$ est une fonction propre associée du problème (V.P.) et vice-versa. 
Maintenant, montrons l'existence de ces points critiques. Pour cela posons

$$
c_{k}=\inf _{K \in A_{k}} \max _{v \in K} \Phi(v)
$$

où

$$
A_{k}=\left\{K \text { compact symétrique de } W_{0}^{1, p}(\Omega) \backslash\{0\} ; \delta(K) \geq k\right\} \quad k \geq 1
$$

$\delta$ étant le genre de Krasnosel'skii.

Lemme 1. Pour $n \geq 1, c_{n}$ défini par 2 est une valeur critique de $\Phi$, de plus nous avons

$$
-\infty<\inf _{W_{0}^{1, p}(\Omega)} \Phi=c_{1} \leq c_{2} \leq \ldots \leq c_{n}<0=\Phi(0)=0
$$

\section{Preuve.}

La suite $\left(A_{k}\right)_{k \geq 1}$ est décroissante donc

$$
-\infty \leq c_{1} \leq c_{2} \leq c_{k} \leq c_{n}
$$

$\Phi$ est paire, de classe $C^{1}$. Pour démontrer ce lemme, nous allons appliquer le théorème fondamental de multiplicité. Il suffit donc que $\Phi$ vérifie les conditions suivantes :

1. $\Phi$ est minorée.

2. $\Phi$ vérifie la condition de Palais-Smale.

3. Pour tout $k \geq 1$, il existe un compact $K$ symétrique de $W_{0}^{-1, p}(\Omega)$ tel que

$$
\delta(K)=k \text { et } \sup _{k} \Phi(v)<0
$$

\section{Verification}

1. Nous avons

$$
\begin{aligned}
p B(u)=\int_{\Omega} m|u|^{p} d x & \leq\|m\|_{\infty} \int_{\Omega}|u|^{p} d x \\
& \leq c_{1}|| m \|_{\infty} \int_{\Omega} \sum_{i=1}^{N}|\nabla u|_{i}^{p} d x \\
& \leq c_{2}|| m \|_{\infty} \int_{\Omega}|\nabla u|^{p} d x \\
& \leq c_{2}\|m\|_{\infty} p A_{p}(u)
\end{aligned}
$$

où $c_{1}$ et $c_{2}$ sont des constantes. 
Ceci grâce à l'inégalité de Sobolev et le fait que la norme $\|\cdot\|_{A_{p}}$ est équivalente à la norme $\|.\|_{1, p} \operatorname{sur} W_{0}^{1, p}(\Omega)$.

Nous avons

$$
\begin{aligned}
p \Phi(u) & =\left(A_{p}(u)\right)^{2}-p B(v) \\
& \geq p\left(A_{p}(u)\right)^{2}-c_{3}\|m\|_{\infty} A_{p}(u)
\end{aligned}
$$

d' où

$$
\left(A_{p}(u)^{2}\right)-B(v) \geq A_{p}(u)\left[A_{p}(u)-\frac{1}{p} c_{3}\|m\|_{\infty}\right]
$$

or $A_{p}$ est coercive et minorée et il en est de même pour $\Phi$, donc

$$
\inf _{W_{0}^{1, p}(\Omega)} \Phi(u)>-\infty
$$

2. Condition (P. S.) Soit $\left(u_{n}\right)_{n}$ une suite dans $W_{0}^{1, p}(\Omega)$ telle que $\Phi\left(u_{n}\right)$ est bornée et $\Phi \prime\left(u_{n}\right) \rightarrow 0$ dans $W^{-1, p \prime}(\Omega)$. $\left(u_{n}\right)_{n}$ est bornée car $\Phi$ est coercive (voir 1.).

Donc, il existe une sous suite notée aussi $\left(u_{n}\right)_{n}$ qui converge faiblement vers $u$ dans $W_{0}^{1, p}(\Omega)$. Donc fortement dans $L^{p}(\Omega)$ et $\left\|u_{n}\right\|_{A_{p}}$ converge vers $\ell \geq 0$.

Si $\ell=0$, i.e. $u_{n} \rightarrow 0$ dans $W_{0}^{1, p}(\Omega)$, (P.S.) est satisfaite.

Si $\ell>0$, il existe $n_{0}$ tel que $\left\|u_{n}\right\|_{A_{p}} \neq 0$ pour $n \geq n_{0}$.

$$
\begin{gathered}
\Phi \prime\left(u_{n}\right)=2 A_{p} \prime(u) A_{p}(u)-B \prime\left(u_{n}\right) \\
A \prime\left(u_{n}\right)=\frac{p}{2} \frac{\Phi \prime\left(u_{n}\right)+m\left|u_{n}\right|^{p-1}-u_{n}}{\|u\|_{A_{p}}} \\
A_{p}^{0}\left(u_{n}\right)=\frac{p}{2} \frac{\Phi \prime\left(u_{n}\right)+m\left|u_{n}\right|^{p-2}-u_{n}}{\|u\|_{A_{p}}}
\end{gathered}
$$

d'où

$$
u_{n}=\left(A_{p}^{0}\right)^{-1} \frac{\Phi \prime\left(u_{n}\right)+m\left|u_{n}\right|^{p-2}-u_{n}}{\|u\|_{A_{p}}}
$$

or $\frac{p}{2} \frac{\Phi \prime\left(u_{n}\right)+m\left|u_{n}\right|^{p-2}-u_{n}}{\|u\|_{A_{p}}}$ converge fortement dans $W^{-1, p \prime}(\Omega)$. La réciproque $\left(A_{p}^{0}\right)^{-1}$ étant continue. Donc $u_{n}$ converge fortement vers $u$ dans $W_{0}^{1, p}(\Omega)$.

3. Soit $n$ un entier tel que $n \geq 1$ et soient $u_{1}, \ldots, u_{n} n$ fonctions définies sur $\Omega$ telles que :

$$
\operatorname{supp}\left(u_{i}\right) \cap \operatorname{supp}\left(u_{j}\right)=\emptyset
$$


et

$$
\int_{\Omega} m\left|u_{i}\right|^{p} d x>0 \text { pour tout } i \in\{1, \ldots, n\}
$$

$u_{i}$ peut être obtenue par approximation dans $L^{2}(\Omega)$ de la fonction caractéristique de l'ensemble

$$
B_{i} \cap\{x \in \Omega ; m(x)>0\}
$$

où $\left(B_{i}\right)_{1 \leq i \leq n}$ sont des boules de $\Omega$ disjointes deux à deux et telles que :

$$
\operatorname{mes}\left(B_{i} \cap\{x \in \Omega ; m(x)>0\}\right) \neq 0
$$

Nous normaliserons les fonctions $u_{i}$ de sorte que $B\left(u_{i}\right)=1$. Soit $F_{n}$ le sous espace de $W_{0}^{1, p}(\Omega)$ engendré par les fonctions $u_{i}$.

Pour tout $v \in F_{n}, v=\sum_{i=1}^{n} \alpha_{i} u_{i}$

$$
B(v)=\sum_{i=1}^{n}\left|\alpha_{i}\right| B\left(u_{i}\right)=\sum_{i=1}^{n}\left|\alpha_{i}\right|^{p}
$$

Ainsi l'application $v \mapsto(B(v))^{1 / p}$ est une norme sur $F_{n}$.

Comme $F_{n}$ est un espace de dimension finie et que toutes les normes sont équivalentes sur un espace de dimension finie, il existe $c$ tel que

$$
c A_{p}(v) \leq B(v) \leq \frac{1}{c} A_{p}(v) \text { pour tout } v \in F_{n}
$$

Soit le compact

$$
K=\left\{v \in F_{n} ; \frac{c^{2}}{4} \leq B(v) \leq \frac{c^{2}}{3}\right\}
$$

Montrons que $\sup _{v \in K} \Phi(v)<0$ :

Soit $v \in K$, nous avons

$$
\Phi(v)=\left(A_{p}(v)\right)^{2}-B(v) \leq \frac{1}{c^{2}}(B(v))^{2}-B(v)
$$

Soit $f:\left[\left[\frac{c^{2}}{4}, \frac{c^{2}}{3}\right] \rightarrow \mathbf{R}\right.$ définie par

$$
f(t)=\frac{t^{2}}{c^{2}}-t
$$


donc

$$
f(t)=\frac{2 t}{c^{2}}-1
$$

et par suite

$$
f \prime(t)=0 \Longleftrightarrow t=\frac{c^{2}}{2}
$$

donc sur $\left[\frac{c^{2}}{4}, \frac{c^{2}}{3}\right] f$ est décroissante. Comme

$$
f(t) \leq f\left(\frac{c^{2}}{4}\right)=-\frac{3 c^{2}}{16}
$$

Il s'en suit que pour tout $v \in K$

$$
\Phi(v) \leq f(t) \leq-\frac{3 c^{2}}{4}<0
$$

D'autre part, comme $F_{n}$ est isomorphe à $\mathbf{R}^{n}$, nous identifions $K$ à une couronne $K^{\prime}$ de $\mathbf{R}^{n}$ telle que $S^{n-1} \subset K^{\prime} \subset \mathbf{R}^{n} \backslash\{0\}$ où $S^{n-1}$ est la sphère unité de $\mathbf{R}^{n}$. Nous avons alors

$$
\delta(K)=n
$$

d'où le lemme.

\section{Lemme 2.}

Nous avons $\lim _{n \rightarrow \infty} c_{n}=0$ où $c_{n}$ est défini par 2 .

\section{Preuve.}

Nous montrons que pour tout $\varepsilon$ il existe $n_{\varepsilon} \geq 1$ tel que pour tout $k \in A_{n_{\varepsilon}}$ avec $k \subset E$

$$
\sup _{v \in k} \Phi(v) \geq-\varepsilon
$$

où

$$
E=\left\{v \in W_{0}^{1, p}(\Omega) ; \Phi(v) \leq 0\right\}
$$

Nous aurons le résultat car $\Phi$ est coercive et $E$ est borné dans $W_{0}^{1, p}(\Omega)$ et en utilisant le fait que $I: W_{0}^{1, p}(\Omega) \rightarrow L^{p}(\Omega)$ est compacte, $I$ est l'injection canonique.

Nous avons pour tout $\eta>0$, il existe un espace $F_{\eta}$ de $L^{p}(\Omega)$ de dimension finie et une application $I_{\eta}: E \rightarrow F_{\eta}$ continue et telle que

$$
\sup _{v \in E}\left\|v-I_{\eta}(v)\right\|_{p} \leq \eta
$$


Posons

$$
\tilde{I}_{\eta}(v)=\frac{1}{2}\left(I_{\eta}(v)-I_{\eta}(-v)\right)
$$

Nous vérifions facilement que pour tout $v \in E, \tilde{I}_{\eta}: E \rightarrow F_{\eta}$ est bien définie (car $E$ est symétrique) impaire continue et satisfait

$$
\sup _{v \in E}\left\|v-I_{\eta}(v)\right\|_{p} \leq \eta
$$

Étant donné maintenant $\varepsilon>0$ puisque $E$ est relativement compacte dans $L^{p}(\Omega)$, il résulte de cette dernière inégalité qu'il existe $n_{\varepsilon}>0$ tel que:

$$
\mid B(v)-B\left(\tilde{I}_{\eta_{\varepsilon}}(v) \mid<\frac{\varepsilon}{2}\right.
$$

pour tout $v \in E$. Soit $\delta_{\varepsilon}>0$ tel que $B(v) \leq \frac{\varepsilon}{2}$ pour $\|v-x\|_{p} \leq \delta_{\varepsilon}$. Donc pour tout $v \in E$ avec

$$
\left\|\tilde{I}_{\eta}(v)\right\|_{p} \leq \delta_{\varepsilon}
$$

Nous avons

$$
B(v) \leq \| B(v)-\left.B\left(\tilde{I}_{\eta_{\varepsilon}}(v)\right)\right|_{p}+B\left(\tilde{I}_{\eta_{\varepsilon}}(v)\right)<\varepsilon
$$

Ce qui implique que pour tout compact symétrique $k$ avec

$$
k \subset E \cap\left\{v \in W_{0}^{1, p}(\Omega) ; B(v) \geq \varepsilon\right\}
$$

Nous avons

$$
\tilde{I}_{\eta_{\varepsilon}}(k) \subset\left\{v \in F_{\eta_{\varepsilon}} ;\|v-\|_{p} \geq \delta_{\varepsilon}\right\}
$$

puisque $\tilde{I}_{\eta_{\varepsilon}}(k)$ est symétrique et compact dans $L^{p}(\Omega)$, il découle de cette dernière inclusion que

$$
\delta^{0}\left(\tilde{I}_{\eta_{\varepsilon}}(k)\right) \leq \operatorname{dim}\left(F_{\eta_{\varepsilon}}\right)
$$

où $\delta^{0}$ est le genre dans $L^{p}(\Omega)$ d'une partie compacte symétrique $K$ I $\subset$ $L^{p}(\Omega)$. Finalement, puisque l'applications $\tilde{I}_{\eta_{\varepsilon}}$ est impaire et continue, il résulte de la définition de $\delta$ et $\delta^{0}$ que

$$
\delta(k) \leq \delta^{0}\left(\tilde{I}_{\eta_{\varepsilon}}(k)\right) \leq \operatorname{dim}\left(F_{\eta_{\varepsilon}}\right)
$$


où $\delta^{0}$ est le genre dans $L^{p}(\Omega)$ d'une partie compacte symétrique $K ı \subset$ $L^{p}(\Omega)$. Finalement, puisque l'applications $\tilde{I}_{\eta_{\varepsilon}}$ est impaire et continue, il résulte de la définition de $\delta$ et $\delta^{0}$ que

$$
\delta(k) \leq \delta^{0}\left(\tilde{I}_{\eta_{\varepsilon}}(k)\right) \leq \operatorname{dim}\left(F_{\eta_{\varepsilon}}\right)
$$

Donc pour tout compact symétrique $K \subset E$ tel que

$$
\delta(k) \geq \operatorname{dim}\left(F_{\eta_{\varepsilon}}\right)+1
$$

Il existe $v_{0} \in k$ tel que

$$
\inf _{v \in k} B(v) \leq B\left(v_{0}\right)<\varepsilon
$$

et par suite, puisque $\Phi(v) \geq-B(v)$ nous avons

$$
\sup _{v \in k} \Phi(v) \geq \inf _{v \in k} B(v)>-\varepsilon
$$

d'où le résultat.

\section{Théorème 3.}

Le problème (V. P.) admet une infinité de valeurs propres positives $\left(\lambda_{n}\right)$ telle que

$$
\lim _{n \rightarrow \infty} \lambda_{n}=+\infty
$$

\section{Preuve.}

Nous avons $\lambda_{n}=\frac{1}{2 \sqrt{-c_{n}}}$, d'après le lemme précédent nous avons le résultat.

\section{Remarque 2.}

1. Si nous remplaçons $m$ par $-m$ dans (V.P.) et que $-m$ satisfait

$$
\operatorname{mes}(\{x \in \Omega ;-m(x)>0\}) \neq 0
$$

le problème (V.P.) admet une suite $\left(\lambda_{-n}\right)_{n}$ de valeurs propres négatives, avec $\lim _{n \rightarrow \infty} \lambda_{-n}=-\infty$. 
2. Soit $\left(\lambda_{n}\right)_{n}$ la suite de valeurs propres donnée par le théorème précédent, c-à-d $\lambda_{n}=1 / 2 \sqrt{-c_{n}}$ où $c_{n}$ est défini par $(2)$.

Nous avons [4].

$$
\lambda_{n}=\inf _{k \in A_{n}}\left(\sup \left\{\mathcal{A}_{p}(v) ; v \in k \text { et } B(v)=1\right\}\right)
$$

En particulier

$$
\lambda_{1}=\inf \left\{\mathcal{A}_{p}(v) ; v \in W_{0}^{1, p}(\Omega) \text { et } B(v)=1\right\}
$$

où $\lambda_{1}$ est la première valeur propre de (V.P.). Elle correspond à la valeur critique $c_{1}$. En effet, soit $\lambda \geq 0$ une valeur propre et soit $u$ une fonction propre associée à $\lambda$ telle que $B(u)=1$. Donc $u$ vérifie en particulier

$$
\mathcal{A}_{p}(u)-\lambda B(u)=0
$$

donc $\lambda=\mathcal{A}_{p}(u)$, or $\mathcal{A}_{p}(u)>0$ car $u \neq 0$ donc $\lambda>0$ (en particulier $\lambda_{1}>0$ ) donc $\lambda \geq \lambda_{1}$ par définition de $\lambda_{1}$.

\section{Simplicité de la première valeur propre}

Dans cette section, nous montrons que la première valeur propre $\lambda_{1}$ est simple et que toute fonction propre associée à $\lambda_{1}$ garde un signe constant.

\section{Théorème 4.}

On a :

i) $\lambda_{1}$ est une valeur propre du problème (V.P.).

ii) $u \not \equiv 0$ est une fonction propre associée à $\lambda_{1}$ si et seulement si :

$$
\mathcal{A}_{p}(u)-\lambda_{1} B(u)=0=\inf _{v \in W_{0}^{1, p}(\Omega)}\left(\mathcal{A}_{p}(v)-\lambda_{1} B(v)\right.
$$

iii) Toute fonction propre associée à $\lambda_{1}$ garde un signe constant. (c-à-d $u>0$ ou bien $u<0$ dans $\Omega$.) 


\section{Preuve.}

L'assertion i) est déjà établie (Voir au dessus).

Pour ii), puisque $\mathcal{A}_{p}$ est f.s.c. et coercive, $B$ est faiblement continue dans $W_{0}^{1, p}(\Omega)$, l'infimum dans la définition de $\lambda_{1}$ est atteint par un élément $u \not \equiv 0$. En tenant compte de la $p$-homogéinité de $\mathcal{A}_{p}$ et $B$ et de la définition de $\lambda_{1}$ nous avons :

$$
\mathcal{A}_{p}(u)-\lambda_{1} B(u)=0=\inf _{v \in W_{0}^{1, p}(\Omega)}\left(\mathcal{A}_{p}(v)-\lambda_{1} B(v)\right)
$$

donc

$$
\mathcal{A}_{p}^{\prime}(u)=\lambda_{1} B^{\prime}(u)
$$

et par suite $u$ est une fonction propre associée à $\lambda_{1}$.

iii) Soit $u$ une fonction propre associée à $\lambda_{1}$. Nous avons par définition $u^{+} \not \equiv 0$ ou $u^{-} \not \equiv 0$. Supposons $u^{+} \not \equiv 0^{1}$. Posons $v=u^{+}$dans (V.P.), nous obtenons

$$
\mathcal{A}_{p}\left(u^{+}\right)-\lambda_{1} B\left(u^{+}\right)=0
$$

donc d'après $(3) u^{+}$est une fonction propre, d'après le principe du maximum [3] $u^{+}>0$ sur $\Omega$ et par suite $u^{+}=u$.

\section{Théorème 5 .}

Supposons que l'hypothèse de régularité.

$$
\text { Toute solution du problème }(\mathrm{VP}) \text { est de classe } \mathcal{C}^{1}(\Omega)
$$

est satisfaite, alors :

$\lambda_{1}$ est une valeur propre simple de $\mathcal{A}_{p}^{0}$.

c-à-d si $u$ et $v$ sont deux fonctions propres associées à $\lambda_{1}$ alors il existe un réel $\alpha$ tel que $u=\alpha v$

\section{Preuve.}

Soient $u$ et $v$ deux fonctions propres positives associées à $\lambda_{1}$. Nous avons $u>$ et $v>0$ (Voir principe du maximum plus haut). De même $\|u\|_{\infty}<+\infty$ et $\|v\|_{\infty}<+\infty$ (Voir estimation $L^{\infty}$ au chapitre 1 ). Soit $\varepsilon>0$, posons

$$
\begin{gathered}
u_{\varepsilon}=u+\varepsilon \quad v_{\varepsilon}=v+\varepsilon \\
\eta(u, v)=\frac{(u+\varepsilon)^{p}-(v+\varepsilon)^{p}}{(u+\varepsilon)^{p-1}}
\end{gathered}
$$




$$
\eta(v, u)=\frac{(v+\varepsilon)^{p}-(u+\varepsilon)^{p}}{(v+\varepsilon)^{p-1}}
$$

\section{Lemme 6.}

La fonction $\eta(u, v)$ est à valeurs dans $W_{0}^{1, p}(\Omega)$.

Preuve.

Soient $\left(u_{n}\right)_{n}$ et $\left(v_{n}\right)_{n}$ deux suites dans $C_{0}^{\infty}$ telles que :

$$
u_{n} \rightarrow u \text { et } v_{n} \rightarrow v \text { pour la norme de } W_{0}^{1, p}(\Omega)
$$

et

$$
\forall n \quad 0 \leq u_{n}, v_{n} \leq M<+\infty
$$

donc

$$
\eta_{n}=\frac{\left(u_{n}+\varepsilon\right)^{p}-\left(v_{n}+\varepsilon\right)^{p}}{\left(u_{n}+\varepsilon\right)^{p-1}} \in W_{0}^{1, p}(\Omega)
$$

La suite $\left(\left\|\eta_{n}\right\|_{1, p}\right)_{n}$ est bornée dans $W_{0}^{1, p}(\Omega)$, donc une sous suite notée aussi $\left(\eta_{n}\right)_{n}$ converge faiblement vers $\omega$ dans $W_{0}^{1, p}(\Omega)$, donc fortement dans $L^{p}(\Omega)$ et presque partout vers $\omega$ dans $\Omega$. D'autre part $\left(\eta_{n}\right)_{n}$ converge presque partout vers $\eta$ dans $\Omega$, donc $\omega=\eta$ et par suite $\eta \in W_{0}^{1, p}(\Omega)$.

\section{Suite de la preuve du théorème :}

Les couples $\left(\lambda_{1}, u\right)$ et $\left(\lambda_{1}, v\right)$ sont solutions du problème (V.P.), donc en particulier ils vérifient :

$$
\int_{\Omega} \sum_{i=1}^{N}|\nabla u|_{i}^{p-2} a_{i}(\nabla u, \nabla \eta(u, v)) d x=\lambda_{1} \int_{\Omega} m(x) u^{p-1} \eta(u, v) d x
$$

et

$$
\int_{\Omega} \sum_{i=1}^{N}|\nabla v|_{i}^{p-2} a_{i}(\nabla v, \nabla \eta(v, u)) d x=\lambda_{1} \int_{\Omega} m(x) v^{p-1} \eta(v, u) d x
$$

Nous avons

$$
\eta(u, v)=\frac{u_{\varepsilon}^{p}-v_{\varepsilon}^{p}}{u_{\varepsilon-1}^{p}}=u_{\varepsilon}-\left(\frac{v_{\varepsilon}}{u_{\varepsilon}}\right)^{p-1} v_{\varepsilon}
$$

donc

$$
\nabla \eta(u, v)=\left\{1+(p-1)\left(\frac{v_{\varepsilon}}{u_{\varepsilon}}\right)^{p}\right\} \nabla u_{\varepsilon}-p\left(\frac{v_{\varepsilon}}{u_{\varepsilon}}\right)^{p-1} \nabla v_{\varepsilon}
$$


De même

$$
\nabla \eta(v, u)=\left\{1+(p-1)\left(\frac{u_{\varepsilon}}{v_{\varepsilon}}\right)^{p}\right\} \nabla v_{\varepsilon}-p\left(\frac{u_{\varepsilon}}{v_{\varepsilon}}\right)^{p-1} \nabla u_{\varepsilon}
$$

Nous avons

$$
\nabla u_{\varepsilon}=\nabla u \quad \text { et } \quad \nabla v_{\varepsilon}=\nabla v
$$

d'où

$$
\begin{aligned}
& \int_{\Omega} \sum_{i=1}^{N}|\nabla u|_{i}^{p-2} a_{i}\left(\nabla u,\left[1+(p-1)\left(\frac{v_{\varepsilon}}{u_{\varepsilon}}\right)^{p}\right] \nabla u_{\varepsilon}+a_{i}\left[\nabla u, p\left(\frac{v_{\varepsilon}}{u_{\varepsilon}}\right)^{p-2} \nabla v_{\varepsilon}\right] d x\right. \\
& \left.=\int_{\Omega} \sum_{i=1}^{N}|\nabla u|_{i}^{p-2}\left[1+(p-1)\left(\frac{v_{\varepsilon}}{u_{\varepsilon}}\right)^{p} a_{i}(\nabla u, \nabla u)-p \frac{v_{\varepsilon}}{u_{\varepsilon}}\right)^{p-2} a_{i}(\nabla u, \nabla v)\right] d x \\
& \left.=\int_{\Omega} \sum_{i=1}^{N}|\nabla u|_{i}^{p-2}\left[1+(p-1)\left(\frac{v_{\varepsilon}}{u_{\varepsilon}}\right)^{p}-p \frac{v_{\varepsilon}}{u_{\varepsilon}}\right)^{p-2}|\nabla u|_{i}^{p-2} a_{i}(\nabla u, \nabla v)\right] d x \\
& =\lambda_{1} \int_{\Omega} m u^{p-1} \eta(u, v) d x \text { car pour } i=1, \ldots, N, a_{i}(\nabla u, \nabla u)=|\nabla u|_{i}^{2} .
\end{aligned}
$$

Donc

$$
\begin{aligned}
& \left.\int_{\Omega} \sum_{i=1}^{N}|\nabla u|_{i}^{p-2}\left[1+(p-1)\left(\frac{v_{\varepsilon}}{u_{\varepsilon}}\right)^{p}-p \frac{v_{\varepsilon}}{u_{\varepsilon}}\right)^{p-2}|\nabla u|_{i}^{p-2} a_{i}(\nabla u, \nabla v)\right] d x \\
& \quad=\lambda_{1} \int_{\Omega} m u^{p-1} \eta(u, v) d x
\end{aligned}
$$

De même, nous avons

$$
\begin{aligned}
& \left.\int_{\Omega} \sum_{i=1}^{N}|\nabla v|_{i}^{p-2}\left[1+(p-1)\left(\frac{u_{\varepsilon}}{v_{\varepsilon}}\right)^{p}-p \frac{u_{\varepsilon}}{v_{\varepsilon}}\right)^{p-2}|\nabla v|_{i}^{p-2} a_{i}(\nabla v, \nabla u)\right] d x \\
& \quad=\lambda_{1} \int_{\Omega} m v^{p-1} \eta(v, u) d x
\end{aligned}
$$

Et par suite en faisant la somme de ces deux dernières équations, nous obtenons

$$
\begin{gathered}
\int_{\Omega} \sum_{i=1}^{N}\left(\left\{1+(p-1)\left(\frac{v_{\varepsilon}}{u_{\varepsilon}}\right)^{p}\right\}|\nabla u|_{i}^{p-2}+1+(p-1)\left(\frac{v_{\varepsilon}}{u_{\varepsilon}}\right)^{p}|\nabla u|_{i}^{p}\right) d x \\
-\int_{\Omega} \sum_{i=1}^{N}\left[1+(p-1)\left(\frac{v_{\varepsilon}}{u_{\varepsilon}}\right)^{p-1}|\nabla u|_{i}^{p-2}(\nabla u, \nabla v)+p\left(\frac{u_{\varepsilon}}{v_{\varepsilon}}\right)\right] d x \\
=\lambda_{1} \int_{\Omega} m\left[\left(\frac{u}{u+\varepsilon}\right)^{p-1}\right]-\left(\frac{v}{v+\varepsilon}\right)^{p-1}
\end{gathered}
$$


Posons

$$
I_{\varepsilon}=\int_{\Omega} m\left[\left(\frac{u}{u+\varepsilon}\right)^{p-1}\right]-\left(\frac{v}{v+\varepsilon}\right)^{p-1} \cdot\left(u_{\varepsilon}^{p}-v_{\varepsilon}^{p}\right.
$$

Nous avons

$$
\nabla \log u_{\varepsilon}=\frac{1}{u_{\varepsilon}} \nabla u_{\varepsilon}=\frac{1}{u_{\varepsilon}} \nabla u
$$

$$
\lambda_{1} I_{\varepsilon}(x)=\sum_{i=1}^{N} \int_{\Omega} u_{\varepsilon}^{p}\left[\left|\nabla \log u_{\varepsilon}\right|_{i}^{p}-\left|\nabla \log v_{\varepsilon}\right|_{i}^{p}-p\left|\nabla \log v_{\varepsilon}\right|_{i}^{p-2}\right.
$$

$\mathrm{a}_{i}\left(\nabla \log u_{\varepsilon}, \nabla u_{\varepsilon}-\nabla \log v_{\varepsilon}\right] d x+\sum_{i=1}^{N} \int_{\Omega} v_{\varepsilon}^{p}\left[\left|\nabla \log v_{\varepsilon}\right|_{i}^{p}-\left|\nabla \log u_{\varepsilon}\right|_{i}^{p}-\right.$ $p\left|\nabla \log u_{\varepsilon}\right|_{i}^{p-2} a_{i}\left(\nabla \log v_{\varepsilon}, \nabla v_{\varepsilon}-\nabla \log u_{\varepsilon}\right] d x$

pour la suite de la démonstration, nous avons besoin du lemme suivant

\section{Lemme 7.}

Pour tout $\xi_{1}, \xi_{2} \in^{N}$ et pour presque tout $x \in \Omega$, nous avons : Si $p \geq 2$

$$
\left|\xi_{2}\right|_{i}^{p} \geq\left|\xi_{1}\right|_{i}^{p}+p\left|\xi_{1}\right|_{i}^{p-2} a_{i}\left(\xi_{1},\left(\xi_{2}-\xi_{1}\right)\right)+\frac{C(p)\left|\xi_{2}-\xi_{1}\right|_{i}^{2}}{\left(2^{p-1}-1\right)^{2-p}}
$$

et si $1<p<2$

$$
\left|\xi_{2}\right|_{i}^{p} \geq\left|\xi_{1}\right|_{i}^{p}+p\left|\xi_{1}\right|_{i}^{p-2} a_{i}\left(\xi_{1},\left(\xi_{2}-\xi_{1}\right)\right)+\frac{C(p)\left|\xi_{2}-\xi_{1}\right|_{i}^{2}}{\left(\left|\xi_{1}\right|_{i}-\left|\xi_{2}\right|_{i}\right)^{2-p}}
$$

où $C(p)>0$ ne dépend pas de $\xi_{1}, \xi_{2}, x \in \Omega$ et $N$.

Proof. [Preuve du lemme] Pour la démonstration de ce lemme, voir [5]. $\square$ Suite de la démonstration du théorème D'après le lemme précédent, nous avons pour $p \geq 2$

$$
\lambda_{1} I_{\varepsilon}(m) \geq C(p) \int_{\Omega} \sum_{i=1}^{N} \frac{\left(u_{\varepsilon}^{p}+v_{\varepsilon}^{p}\right.}{\left(2^{p-1}-1\right)} \cdot\left|\nabla \log u_{\varepsilon}-\nabla \log v_{\varepsilon}\right|_{i}^{p} d x
$$

et pour tout $1<p<2$

$$
\lambda_{1} I_{\varepsilon}(m) \geq C(p) \int_{\Omega} \sum_{i=1}^{N} \frac{\left(u_{\varepsilon}^{p}+v_{\varepsilon}^{p}\right)\left|\nabla \log u_{\varepsilon}-\nabla \log v_{\varepsilon}\right|_{i}}{\left(\left|\nabla \log u_{\varepsilon}-\nabla \log v_{\varepsilon}\right|_{i}\right)^{p-2}} d x
$$


Puisque

$$
\nabla \log u_{\varepsilon}=\frac{1}{u_{\epsilon}} \nabla u \quad \text { et } \quad \nabla \log v_{\varepsilon}=\frac{1}{v_{\epsilon}} \nabla v
$$

Alors pour $p \geq 2$, nous avons

$$
\lambda_{1} I_{\varepsilon}(m) \geq \frac{C(p)}{\left(2^{p-1}-1\right)} \sum_{i=1}^{N} \int_{\Omega}\left[\frac{1}{(u+1)^{p}}+\frac{1}{(v+1)^{p}}\right] \cdot\left|u_{\varepsilon} \nabla v-v_{\varepsilon} \nabla u\right| d x \geq 0
$$

et pour $1<p<2$ nous avons

$$
(4.5) \lambda_{1} I_{\varepsilon}(m) \geq C(p) \int_{\Omega} \sum_{i=1}^{N} \frac{\left(u_{\varepsilon} \cdot v_{\varepsilon}\right)\left(u_{\varepsilon}^{p}+v_{\varepsilon}^{p}\right)\left|v_{\varepsilon} \nabla u-u_{\varepsilon} \nabla v\right|_{i}^{2}}{\left(v_{\varepsilon}\left|\nabla u_{\varepsilon}\right|_{i}+u_{\varepsilon}\left|\nabla v_{\varepsilon}\right|_{i}+1\right)^{2-p}} d x \geq 0
$$

Comme les suites $\left(u_{\varepsilon}\right)$ et $\left(v_{\varepsilon}\right)$ convergent uniformément vers $u$ et $v$ quand $\varepsilon \rightarrow 0$ et par application du lemme de Fatou nous obtenons : Pour $p \geq 2$

$$
\begin{gathered}
\lambda_{1} \lim _{\varepsilon \rightarrow 0} I_{\varepsilon} \geq \frac{1}{2^{p-1}-1} \sum_{i=1}^{N} \int_{\Omega}\left[\frac{1}{(u+1)^{p}}+\frac{1}{(v+1)^{p}}\right] . \\
|u \nabla v-v \nabla u|_{i}^{p} \geq 0
\end{gathered}
$$

et pour $1<p<2$

$$
\lambda_{1} \lim _{\varepsilon \rightarrow 0} I_{\varepsilon} \geq C(p) \sum_{i=1}^{N} \int_{\Omega} \frac{u \cdot v\left(u^{p}+v^{p}\right)|u \nabla v-v \nabla u|_{i}^{2}}{\left(v|\nabla u|_{i}+u|\nabla v|_{i}+1\right)^{2-p}} d x \geq 0
$$

Montrons que

$$
\lim _{\varepsilon \rightarrow 0} I_{\varepsilon}(m)=0
$$

Nous avons

$$
\begin{gathered}
I_{\varepsilon}(m)=\int_{\Omega} m u^{p-1} u_{\varepsilon} d x+\int_{\Omega} m v^{p-1} v_{\varepsilon} d x-\int_{\Omega} m\left(\frac{v}{v_{\varepsilon}} \cdot u_{\varepsilon}\right)^{p-1} u_{\varepsilon} d x- \\
\int_{\Omega} m\left(\frac{u}{u_{\varepsilon}} \cdot v_{\varepsilon}\right)^{p-1} v_{\varepsilon} d x \text { Puisquem } \in L^{\infty}(\Omega), \text { grâce au théorème de la } \\
\text { convergence dominée nous obtenons }
\end{gathered}
$$

$$
\lim _{\varepsilon \rightarrow 0} I_{\varepsilon}(m)=0
$$


et d'après (4.6) et (4.7) nous avons

$$
\sum_{i=1}^{N}|v \nabla u-u \nabla v|_{i}=0
$$

et puisque $\frac{u}{v} \in L^{\infty}$ (d'après le principe du maximum), nous avons $\nabla\left(\frac{u}{v}\right)=0$, donc il existe $k>0$ tel que

$$
u=k v
$$

d'où le résultat.

\section{Isolation de la première valeur propre}

Nous montrerons d'abord que toute fonction propre associée à une valeur propre positive autre que la première valeur propre change de signe. Ensuite, nous montrerons que $\lambda_{1}$ est isolée.

Théorème 8. Si $v$ est une fonction propre associée à une valeur propre $\lambda>0$ telle que $\lambda \neq \lambda_{1}$ alors $v$ change de signe, c-à-d $v^{+} \not \equiv 0$ et $v^{-} \not \equiv 0$ dans $\Omega$, de plus nous avons

$$
\min \left\{\operatorname{mes}\left(\Omega^{+}\right), \text {mes }\left(\Omega^{-}\right)\right\} \geq\left(\lambda\|m\|_{\infty} C^{p}\right)^{\sigma}
$$

où

$$
\Omega^{+}=\{x \in \Omega ; v(x)>0\} \quad \Omega^{-}=\{x \in \Omega ; v(x)<0\}
$$

et $C$ est une constante indépendante de $v$ et de $\lambda$, et $\sigma=-N / p$ si $p<N$ et $\sigma=-2$ si $p \geq N$.

Proof. [Preuve] Soit $u$ une fonction propre associée à $\lambda_{1}$ telle que

$$
\int_{\Omega} \sum_{i=1}^{N}|\nabla u|_{i}^{p}=1
$$

Supposons qu'il existe une valeur propre $\lambda>0$ telle que $\lambda \neq \lambda_{1}$ ayant une fonction propre $v \geq 0$ vérifiant

$$
\int_{\Omega} \sum_{i=1}^{N}|\nabla v|_{i}^{p}=1
$$


En particulier nous avons

$$
\int_{\Omega} \sum_{i=1}^{N}|\nabla u|_{i} a_{i}(\nabla u, \nabla \eta(u, v)) d x=\lambda_{1} \int_{\Omega} m u^{p-1} \eta(u, v) d x
$$

et

$$
\int_{\Omega} \sum_{i=1}^{N}|\nabla v|_{i} a_{i}(\nabla v, \nabla \eta(v, u)) d x=\lambda_{1} \int_{\Omega} m v^{p-1} \eta(v, u) d x
$$

avec

$$
\eta(u, v)=\frac{(u+\varepsilon)^{p}-(v+\varepsilon)^{p}}{(u+\varepsilon)^{p-1}} \text { et } \eta(v, u)=\frac{(v+\varepsilon)^{p}-(u+\varepsilon)^{p}}{(v+\varepsilon)^{p-1}}
$$

La somme de (5.2) et de (5.3) nous donne

$$
J_{\varepsilon}=\int_{\Omega} m\left[\lambda_{1}\left(\frac{u}{u_{\varepsilon}}\right)^{p-1}-\lambda\left(\frac{v}{v_{\varepsilon}}\right)^{p-1}\right]\left(u_{\varepsilon}^{p}-v_{\varepsilon}^{p}\right) d x \geq 0
$$

et

$$
\lim _{\varepsilon \rightarrow 0} J_{\varepsilon}(m)=\left(\lambda_{1}-\lambda\right) \int_{\omega} m\left(u^{p}-v^{p}\right) d x \geq 0
$$

Il s'en suit que :

$$
\left(\lambda_{1}-\lambda\right)\left(\frac{1}{\lambda_{1}} \int_{\Omega} \sum_{i=1}^{N}|\nabla u|_{i}^{p} d x-\frac{1}{\lambda} \int_{\Omega} \sum_{i=1}^{N}|\nabla v|_{i}^{p} d x\right) \geq 0
$$

donc

$$
\left(\lambda_{1}-\lambda\right)\left(\frac{1}{\lambda_{1}}-\frac{1}{\lambda}\right) \geq 0
$$

donc $\lambda=\lambda_{1}$, ce qui est absurde. Donc $v$ change de signe.

Montrons maintenant l'estimation (5.1). pour cela nous remplaçons dans (V.P.), $v$ par $v^{+}$, puis par $v^{-}$. L'inégalité de Hölder nous donne

$$
\left\|v^{+}\right\|_{p}=\lambda \int_{\Omega} m\left|v^{+}\right|^{p} d x \leq \lambda|| v^{+} \|_{p^{*}}^{p}\left(\left(\Omega^{+}\right)\right)^{1-p / p^{*}}
$$

Puis par l'inégalité de Sobolev

$$
\left\|v^{+}\right\|_{1, p^{*}} \leq\left\|\nabla v^{+}\right\|_{1, p}
$$


et en utilisant le fait que $\left(\Omega^{+}\right) \neq 0$ car $v^{+} \not \equiv 0$, nous obtenons

$$
\operatorname{mes}\left(\Omega^{+}\right) \geq\left(\lambda\|m\|_{\infty} C^{p}\right)^{\sigma}
$$

De même pour $v^{-}$. Ce qui nous donne l'estimation (5.1).

Théorème 9. La valeur propre $\lambda_{1}$ est isolée, c-à-d $\lambda_{1}$ est l'unique valeur propre du problème (V.P.) dans un certain intervalle $[0, a]$ où $a>\lambda_{1}$.

Proof. [Preuve] Soit $(\lambda, u)$ une solution du problème (VP). Il est clair que si $\lambda=0, \lambda$ n'est pas une valeur propre.

Par définition de $\lambda_{1}$, nous avons

$$
\|v\|_{\mathcal{A}_{p}}^{p} \geq \lambda_{1} \int_{\Omega} m|v|^{p} d x
$$

D'autre part, nous avons

$$
\|v\|_{\mathcal{A}_{p}}^{p}=\lambda \int_{\Omega} m|v|^{p} d x
$$

d'où

$$
\lambda_{1} \leq \lambda
$$

donc $\lambda_{1}$ est isolée à gauche.

Supposons maintenant qu'il existe une suite $\left(\lambda_{n}\right)_{n},\left(\lambda_{n} \neq \lambda_{1}\right)$ de valeurs propres qui converge vers $\lambda_{1}$.

Soit $u_{n}$ la fonction propre associée à $\lambda_{n}$ telle que $\left\|u_{n}\right\|_{1, p}=1$ pour tout $n$. Pour une sous suite encore notée $\left(u_{n}\right)_{n},\left(u_{n}\right)_{n}$ converge faiblement dans $\Omega$ vers une fonction $u \in W_{0}^{1, p}$.

Comme:

$$
u_{n}=\left(\mathcal{A}_{p}^{0}\right)^{-1}\left(\lambda_{n} m(x)\left|u_{n}\right|^{p-2} u_{n}\right)
$$

et puisque $\left(\lambda_{n} m(x)\left|u_{n}\right|^{p-2} u_{n}\right)$ converge fortement dans $L^{p^{\prime}}(\Omega)$ donc dans $W^{-1, p^{\prime}}(\Omega)$ et $\left(\mathcal{A}_{p}^{0}\right)^{-1}: W^{-1, p^{\prime}} \rightarrow W_{0}^{1, p}$ est continue.

Donc $\left(u_{n}\right)_{n}$ converge fortement dans $W_{0}^{1, p}$ vers $u$ avec $u$ une fonction propre de norme 1 associée à $\lambda_{1}$, puisque $u$ garde un signe constant (Voir §précédent.), supposons $u>0$ par exemple.

Soit $\varepsilon>0 u$ étant strictement positive, donc il existe un réel $\eta_{\varepsilon}>0$ et un ensemble $\Omega_{\varepsilon} \subset \Omega$ avec mes $\left(\Omega \backslash \Omega_{\varepsilon}\right) \leq \frac{\varepsilon}{2}$ et $u(x) \geq 2 \eta_{\varepsilon}$ 
pour tout $x \in \Omega_{\varepsilon}$. et comme $\left(u_{n}\right)_{n}$ converge presque partout dans $\Omega$ vers $u$, par le théorème d'Egorov, nous avons la suite $\left(u_{n}\right)_{n}$ qui converge fortement vers $u$ uniformément à l'extérieur d'un ensemble de mesure arbitrairement petite, donc il existe un entier $N_{\varepsilon}$ et un ensemble mesurable $\Omega_{\varepsilon}^{\prime} \subset \Omega$ avec

$$
\operatorname{mes}\left(\Omega \backslash \Omega_{\varepsilon}^{\prime}\right) \leq \frac{\varepsilon}{2}
$$

tels que $\left|u_{n}(x)-u(x)\right| \leq \eta_{\varepsilon}$

Pour tout $x \in \Omega_{\varepsilon}^{\prime}$ et $\eta \geq N_{\varepsilon}$ nous avons

$$
u_{n}(x) \geq \eta_{\varepsilon}>0
$$

pour tout $x \in \Omega_{\varepsilon} \cap \Omega_{\varepsilon}^{\prime}$ et $n \geq N_{\varepsilon}$, en particulier pour tout $n \geq N_{\varepsilon}$, nous avons

$$
\operatorname{mes}\left(\left\{x \in \Omega ; u_{n}(x)>0\right\}\right) \geq \operatorname{mes}\left(\Omega_{\varepsilon} \cap \Omega_{\varepsilon}^{\prime}\right) \geq \operatorname{mes}(\Omega)-\varepsilon
$$

or d'après l'estimation (5.1)

$$
\operatorname{mes}\left(\left\{x \in \Omega ; u_{n}(x)>0\right\}\right) \geq\left(\|m\|_{\infty} C^{p} \sup \left(\lambda_{n}\right)\right)^{\sigma}
$$

Si nous prenons $\varepsilon=\frac{1}{2}\left(\varepsilon^{p}\|m\|_{\infty}\right)^{\sigma}$ nous aboutissons à une contradiction. 


\section{References}

[1] A. Anane Étude des valeurs propres et de la croissance pour l'opérateur $p$-Laplacien, Thèse de doctorat, Université Libre de Bruxelles, 1987.

[2] H. Brezis et F. Browder, Sur une propriété des espaces de Sobolev, C.R.A.S. Paris, 287, 113-115, 1998.

[3] A. Benazzi, Thèse d'état, Université Mohamed I, Oujda, en préparation.

[4] O. Chakrone, Spectre d'ordre supérieur dans des problèmes aux limites quasilinéaires, et sur un théorème de points critique et application à un problème de non-résonance entre deux valeurs propres du $p$-Laplacien, Thèse d'état, Université Mohamed I, Oujda, 1998.

[5] P. Lindqvist, On the equation $\operatorname{div}\left(|\nabla u|^{p-2} \nabla u\right)+\lambda u^{p-2} u=0$ Prog. A.M.S., 1, 109, 1960

Received : March, 2000.
A. Anane
and
O. Chakrone
Département de mathématiques
Faculté des sciences
Université Mohamed I
Oujda - Maroc
e-mail : anane@sciences.univ-oujda.ac.ma
A. Benazzi
Département génie électrique
École supérieure de technologie
Oujda - Maroc
e-mail : benazzi@est.univ-oujda.ac.ma 